\title{
Legitimidad del ejercicio versus la de origen: análisis del primer año de gestión de las últimas tres alcaldías capitalinas
}

Legitimacy of the Exercise Versus Legitimacy of Origin: Analysis of Last Three Capital City Mayors' Managements

A legitimidade do exercício versus a de origem: análise da gestão das três últimas prefeituras da capital

\author{
Guido Moncayo Vives \\ Universidad Nacional del Litoral. Santa Fe, Argentina \\ guidomoncayo@gmail.com \\ https://orcid.org/0000-0001-8186-2427
}

DOI: https://doi.org/10.32719/25506641.2021.10.8

Recibido: 27 de abril de 2020 - Revisado: 26 de junio de 2020

Aceptado: 3 de agosto de 2020 • Publicado: 1 de julio de 2021

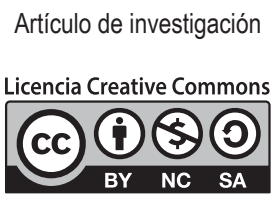




\section{Resumen}

La presente investigación busca comparar el primer año de gestión y sus niveles de legitimidad de los último tres alcaldes de Quito y la actual administración municipal en la ciudad de Guayaquil, para analizar comparativamente los niveles de legitimidad de origen o de elección respecto a la alcanzada luego del primer año de gestión y, de presentarse diferentes niveles de legitimidad, poder identificar cuáles han sido las acciones o las estrategias ejecutadas para que la legitimidad del ejercicio prime sobre la de origen. Es así que el artículo en su primera parte empieza con un marco conceptual que analizará conceptos como los sistemas de partidos, sistemas de gobierno, regímenes políticos, sistemas electorales y la legitimidad con sus diferentes tipos, seguido por una sección donde se analiza la elección y gestión de anteriores alcaldes capitalinos y la actual alcaldesa guayaquileña y se cierra con la tercera parte que buscará analizar la gestión de las tres alcaldías antes mencionadas en comparación con el desempeño de la actual alcaldía quiteña. Se realiza este artículo a partir de una metodología histórico-analítica, con análisis de datos cuantitativos y cualitativos, encontrando como principal limitante la diferencia en algunos casos de la temporalidad de los datos de los exalcaldes, que para el propósito de este estudio no se considera relevante.

Palabras clave: administración pública, gobierno local, políticas públicas, gestión pública, COVID-19.

JEL: D78 Análisis positivo de las decisiones políticas y de su ejecución.

\section{Abstract}

The present investigation seeks to compare the first year of administration and its levels of legitimacy of the last three mayors of Quito and the current municipal administration in the city of Guayaquil, to comparatively analyze the levels of legitimacy of origin or election with respect to that achieved later of the first year of management, and of presenting different levels of legitimacy, to be able to identify what have been the actions or the strategies implemented so that the legitimacy of the exercise prevails over that of origin? Thus, the article in its first part begins with a conceptual framework that will analyze concepts such as party systems, government systems, political regimes, electoral systems and legitimacy with its different types, followed by a section where the election and management of previous capital mayors and the current mayor of Guayaquil and closes with the third part that will seek to analyze the management of the three aforementioned mayoralties compared to the performance of the current Quito mayor. This article is made from a historical analytical methodology, with analysis of quantitative and qualitative data, finding as the main limitation the difference in some cases of the temporality of the data of former mayors, which for the purpose of this study I consider not has been relevant.

Keywords: Public administration, local government, public policy, public management, COVID-19.

JEL: D78 Positive analysis of policy formulation and implementation. 


\section{Resumo}

A presente pesquisa busca comparar o primeiro ano de gestão e seus níveis de legitimidade dos três últimos prefeitos de Quito (2009-2010, 2014-2015 e 2019-2020) e a atual administração municipal da cidade de Guayaquil (2019-2020), para analisar comparativamente os níveis de legitimidade de origem ou de eleição no que diz respeito àquela alcançada no primeiro ano de gestão e, caso se apresentem diferentes níveis de legitimidade, identificar quais foram as ações ou estratégias para que a legitimidade do exercício prevalecesse sobre a de origem. Na parte conceitual, analisam-se os conceitos de sistemas de partidos, sistemas de governo, regimes políticos, sistemas eleitorais e a legitimidade, com seus diferentes tipos, seguida de uma seção onde se analisa a eleição e a gestão de prefeitos anteriores da capital e da atual prefeita de Guayaquil. Finalmente, faz-se uma análise da gestão das três prefeituras previamente mencionadas em comparação com a atual prefeitura de Quito, a qual teve, no último ano, que enfrentar a crise sanitária provocada pelo Covid-19. A metodologia é histórico-analítica, com observação de dados quantitativos e qualitativos, tendo como principal conclusão o fato de que a forma de gerir o público exerce influência sobre as variações entre a legitimidade de origem e a de exercício.

Palavras-chave: Administração pública, governo local, políticas públicas, gestão pública, COVID-19.

JEL: D78 Análise positiva das decisões políticas e sua execução.

\section{Introducción}

( e busca investigar si durante su casi primer año de gestión, el alcalde de la capital ecuatoriana, Jorge Yunda Machado, ha podido superar la deficiente legitimidad con la que arrancó su mandato, con apenas un quinto de los electores, en medio de críticas por su inexperiencia en la administración pública y con el gran reto de sacar adelante a una ciudad que en los últimos años había caído en una especie de estancamiento social, económico y político. Además, se trata de responder a la pregunta de cuáles son los aspectos que han permitido al alcalde Yunda crecer en sus niveles de popularidad en un año de gestión, es decir, qué ha hecho de diferente Yunda con respecto a otros alcaldes de la ciudad y del país — pasados y actuales-, para aumentar de forma radical su legitimidad del ejercicio con relación a su legitimidad de origen.

Se parte de un marco conceptual que analiza conceptos como los sistemas de partidos, sistemas de gobierno, regímenes políticos, sistemas electo- 
rales y su evolución en el tiempo hasta llegar al siglo XXI, con las TIC a la vanguardia, que influyen fuertemente en la forma de hacer política, incluso en países en vías de desarrollo como el Ecuador, donde la brecha digital aún es amplia, pero con tendencia a la baja. En una segunda parte, se analiza la experiencia de los exalcaldes del Distrito Metropolitano de Quito (DMQ) Augusto Barrera y Mauricio Rodas con relación a su legitimidad de origen y del ejercicio, con el fin de analizar cuál ha sido el comportamiento con respecto a las actuales administraciones locales, tanto en el DMQ como en Guayaquil.

\section{Marco conceptual}

\section{Desde los sistemas de partidos a las legitimidades democráticas}

Los sistemas políticos contemporáneos se desarrollan a partir de dinámicas que, hasta hace unos años, eran impensadas. Entre ellas, las marcadas por el vertiginoso crecimiento de la influencia de las tecnologías de la información y las comunicaciones en la sociedad, en su intención del voto y en cómo los políticos se mueven para gestionar de la mejor manera ese capital político son, posiblemente, las más importantes.

Por lo que, para comprender de mejor manera el contexto en el que se analizará el primer año de gestión del alcalde del DMQ, Dr. Jorge Yunda Machado, se hace imperante el abordar ciertos conceptos que darán luces al respecto. El primero de ellos es el concerniente al sistema de partidos, que de forma general ha tenido una evolución a la par con "la democracia, la ampliación del sufragio universal y las prerrogativas parlamentarias" (Duverger 1957, 15).

El sistema de partidos está determinado por el régimen político, el cual es definido por Duverger $(1962,13)$ como el "conjunto de instituciones políticas que funcionan en un país determinado y en un momento determinado; en cierto modo, los regímenes políticos son constelaciones cuyas instituciones políticas son las estrellas". Los sistemas de partidos son prácticamente universales (Malamud 2003) y la forma como están constituidos en un país determina, de manera importante, cómo se desarrollan las elecciones. Gene- 
ralmente existen tres tipos de sistemas de partidos: el partido único (como en China o Cuba), el bipartidismo (como se da tácitamente en los Estados Unidos) y el multipartidismo, como en el caso ecuatoriano (Duverger 1962).

Otro concepto relevante es el relacionado a los sistemas electorales. En la actualidad, los sistemas electorales presuponen a la designación por elección como "la única natural y legítima, puesto que un país es considerado democrático cuando sus gobernantes son elegidos por elecciones lo más libres y sinceras posibles" (79). El contar, en la actualidad, con sufragio universal es el resultado de largas luchas durante el siglo XIX, donde se buscaba ampliar el derecho al voto, hasta entonces reservado a unos cuantos, hacia la totalidad de la ciudadanía (Duverger 1962).

El devenir histórico de la democracia ha tendido siempre - de forma natural - hacia el sufragio universal. Sin embargo, este hecho, aunque se vea adecuado, "ha parecido durante mucho tiempo como peligroso (y siempre lo parece) a los grupos sociales cuyas prerrogativas políticas o cuyos privilegios económicos se encontraban amenazados por el poder así concedido a las masas populares" (84). Y es que, "en la teoría clásica, los elegidos son considerados como los representantes de los electores en el sentido jurídico del término, es así que se considera a los electores como los mandantes y a los elegidos como mandatarios" (113), hecho que, por lo antes mencionado, no agrada a los grupos de poder fácticos.

Una regla fundamental en los sistemas electorales democráticos es el principio de mayoría. Bajo este principio, se entiende que cualquier persona que vota con la mayoría (y esto lo hace la gran parte de los votantes) gana, mientras quien vota con la minoría, no solo que pierde, sino que desperdicia su voto. Este hecho delinea el pensamiento lógico que quien votó por la minoría debe someterse a la mayoría, cayendo en lo que se conoce como la tiranía de la mayoría, considerando que inclusive, muchas veces, la mayoría es solo la minoría mayor (Sartori 1995).

Por otro lado, el sistema de gobierno es un factor a tener en cuenta al momento de escudriñar la legitimidad en cierta circunstancia electoral. A nivel general, se destacan esencialmente "tres tipos de órganos gubernamentales: los individuos, los comités y las asambleas. El poder puede ser ejercido por un hombre, por un pequeño grupo de hombres o por una gran asamblea de hombres" (Duverger 1962, 138). En el caso de Ecuador, se cuenta con un sis- 
tema de gobierno presidencialista, unitario y mayoritariamente centralizado en sus competencias principales. Las ciudades son gobernadas por alcaldes que, al igual que el presidente de la república, son designados por elección popular (EC 2008, art. 253).

Con relación al concepto de legitimidad, que se constituye en el eje central del presente documento, tratando de encontrar un concepto mínimo de legitimidad, Linz $(1991,38)$ la define como "la creencia de que, a pesar de sus limitaciones y fallos, las instituciones políticas existentes son mejores que otras que pudieran haber sido establecidas, y que por tanto pueden exigir obediencia”.

Por otro lado, para Duverger (1962) la legitimidad parte de principios como la legalidad, la soberanía y el deber moral; un poder que no contenga en sí mismo estos principios es ilegítimo y no es digno de obediencia, pero a su vez, si los posee en un momento y en un espacio definidos, ese gobierno es inquebrantable. Adicionalmente, se trae a colación otro factor que debe contener un gobierno para ser legítimo políticamente, y es la eficiencia administrativa; es así como en palabras de García $(2000,145)$ "los gobiernos no solo pecan por exceso, sino también por defecto".

Es importante revisar algunas taxonomías que a través del tiempo se han concedido al concepto de legitimidad. Una muy importante y tal vez la más conocida es la de Weber $(2002,172)$, quien enuncia tres tipos de legitimidad: la legal que proviene de la norma y el derecho, la tradicional que viene justamente de las tradiciones y de cómo se han hecho las cosas durante mucho tiempo y, finalmente, la carismática que surge de la "entrega extra cotidiana a la santidad, heroísmo o ejemplaridad de una persona y a las ordenaciones por ella creadas o reveladas".

Otra forma de clasificar los tipos de legitimidad es en relación a la distancia del gobierno respecto a los ciudadanos. Por un lado, se puede identificar una legitimidad de reconocimiento o diferenciación, que justamente "sostiene que la elección de un representante se refiere a un principio de distinción (una selección de los 'mejores')" (Rosanvallon en Annunziata 2011, 397) y, por otro, la legitimidad de proximidad o identidad, que se fortalece a medida que la primera se debilita, convirtiéndose en una forma de relacionamiento entre representantes y representados, promoviendo un lazo político entre ellos y que trasciende a su carácter instituyente (Annunziata 2011). 
En este mismo sentido, está la legitimidad formal, que "deviene del orden político y que comprende aspectos procedimentales relacionados con la forma en que los individuos llegan a detentar el poder político, es decir, el ejercicio electoral en sí, en un sistema democrático" (Rúa 2013, 97), misma que se puede denominar como legitimidad de origen, que es la ganada en las urnas en los procesos electorales que se celebran generalmente en Estados democrcáticos (Prado 2010).

La otra es la legitimidad material, "que se refiere al ejercicio del poder en sí, y que está orientada al cumplimiento de los objetivos definidos para el cargo al cual fueron elegidos" (Rúa 2013, 97), considerándose también con el nombre de legitimidad del ejercicio, misma que se puede fortalecer respecto a la de origen o, por el contrario, se puede ver degradada o desgastada en el tiempo. Este dilema es el que generalmente los políticos que han sido elegidos de forma democrática experimentan, y es el eje central de análisis del presente artículo.

Es importante considerar que autores como Duverger $(1962,38)$ han planteado el hecho de que el concepto de soberanía ligado al de legitimidad evoluciona o se transforma en el tiempo:

Por ejemplo, aún hoy, ciertos pueblos siguen aferrados al derecho divino, la mayoría de las naciones occidentales creen en la soberanía nacional, la URSS y las democracias populares se ligan a la soberanía proletaria, pero siempre lo que en un territorio y momento histórico dado bajo la doctrina de la soberanía que es aceptada por la mayoría es considerado legítimo.

Precisamente la evolución del concepto de legitimidad democrática ha dado fuerza al reconocimiento de otro término vinculado: la legitimación. La legitimación se convierte en el baluarte de los gobiernos que desean mantener o aumentar su legitimidad, y esta se incrementa solo cuando estos tienen el interés de hacerlo, es decir, cuando desde las esferas gubernamentales se efectúan acciones que dan confianza a la ciudadanía de que sus gobernantes están haciendo las cosas bien (Linz 1991; Lipset 2001; Galindo 2016).

En esta línea, y considerando el soporte que los gobiernos requieren para legitimarse, podemos diferencias dos tipos de apoyo. Por un lado, está el apoyo difuso que tiene que ver con el conjunto de valores y principios que hacen parte del imaginario colectivo social y que hacen que los represen- 
tantes que han sido elegidos gobiernen, pero por otro lado está el apoyo específico, que consiste en la realización concreta de estos principios y en la materialización de las atribuciones con que cuentan los gobernantes y para las cuales se los ha elegido (Payne y Allamand 2006).

Las acciones realizadas de manera adecuada por los gobernantes durante su ejercicio generan confianza en la ciudadanía. Esta confianza es el combustible que echa a andar el aparato gubernamental, especialmente en los regímenes relativamente jóvenes, que necesitan darse a conocer como eficientes, eficaces y legítimos (Hernández 2004; Payne y Allamand 2006; Moncagatta, Moscoso y Pachano 2020). El hacer las cosas bien y en el momento preciso, generando resultados (García 2000), es lo que permitirá a los gobiernos ganar la confianza de los ciudadanos, la cual "es esencial para la cohesión social y el bienestar, incluida la reducción de la desigualdad, ya que afecta la capacidad del gobierno para implementar reformas" (OCDE 2020, 26).

Desde hace algún tiempo ya, la legitimidad democrática se está transformando. Ya no se les cree a los partidos políticos tradicionales y la ciudadanía busca reflejarse en sus representantes, votando por "hombres comunes y que compartan con los ciudadanos sus preocupaciones y problemas cotidianos, prestando atención a la singularidad de sus experiencias" (Annunziata 2011, 397). En el caso que se está analizando, se trata de un político relativamente nuevo, que llega a la alcaldía del DMQ con algo más de un quinto de la votación y que desde el inicio de su mandato tuvo grandes cuestionamientos con respecto a su legitimidad, si bien la elección cuenta con la legalidad necesaria, la primera aún está por confirmarse.

Pero aquí entra un factor de análisis decisivo y que es el principal para la tercera parte de este estudio: la legitimidad del ejercicio a partir del apoyo a la gestión con el pasar del tiempo, ya que "en general se asume que la legitimidad debe medirse a través de las percepciones de los ciudadanos, mediante encuestas de opinión pública" (Vairo 2012, 46). Es un problema no menor el llegar a una alcaldía con tan poco electorado, ya que "una baja legitimidad en función del caudal electoral podría dificultarle la conducción eficaz del gobierno y llevar al estancamiento (Payne y Allamand 2006, 22). Esto también lo corrobora Duverger (1962) cuando atribuye al conformismo ser una fuente fundamental de la obediencia al poder, porque hay presión del grupo social a mantener la legitimidad de las urnas, "aunque la victoria 
electoral haya sido con la quinta parte del electorado" (Duverger 1962, 19). Definitivamente, el reto del alcalde Yunda, cuando ganó las elecciones, fue más grande de lo que nadie hubiera pensado, y se intentará analizar si su respuesta para alcanzar una legitimidad completa ha sido la óptima.

\section{Análisis vertical y horizontal}

\section{Revisando alcaldías quiteñas anteriores y la actual alcaldía de Guayaquil}

Las dos elecciones anteriores a la de Jorge Yunda para la alcaldía del DMQ manifestaron cómodas victorias, o lo que en el marco conceptual se ha denomina como la legitimidad formal o de origen. Esta sección revisa las circunstancias en las que se dieron estas dos elecciones, es decir, cómo llegaron a la alcaldía capitalina Augusto Barrera, en 2009 y Mauricio Rodas, en 2014. Las dos elecciones presentan aspectos comunes entre sí, pero también algunos marcadamente diferentes.

Las elecciones generales para alcaldes llevadas a cabo en Ecuador el 26 de abril de 2009 dieron por ganador a Augusto Barrera, candidato por el Movimiento Patria Altiva y Soberana - partido que ostentaba el poder gubernamental desde 2007-. Estas elecciones precisamente se realizaron en medio de un proceso de transformación estatal iniciado por el aquel entonces presidente de la República, Rafael Correa Delgado, quien había llegado al poder dos años atrás con la promesa de gobernar sin el apoyo de los partidos políticos de siempre, sin una lista de por medio de candidatos al congreso y con la propuesta de instaurar una asamblea constituyente que refunde la patria (Freidenberg 2011).

En este contexto social y político, Barrera llega apoyado, sin duda alguna, con uno de los espaldarazos más contundentes que algún candidato a la alcaldía quiteña podía aspirar, ganando con el $43,14 \%$ de los votos y con nueve de los quince concejales, es decir, el $60 \%$ de total. Antonio Ricaurte, de la alianza MMIN-VIVE fue el segundo en las votaciones detrás de Barrera, con el $27,02 \%$, una diferencia de más de $16 \%$. En términos simples, el 
triunfo de Barrera se constituyó en un arranque de labores marcado por una amplia legitimidad de origen, es decir, un amplio apoyo ciudadano.

Tres años más tarde, para mediados de junio de 2012, la empresa encuestadora Perfiles de Opinión, a partir de una encuesta representativa a 2449 personas, reveló que el alcalde del DMQ, Augusto Barrera, mantenía una aprobación a su gestión del 72,6\% (61,8\% de los encuestados cree que es buena y el 10,8\% considera que es muy buena). A esto se suma también que a la pregunta 'En comparación con 12 meses atrás, ¿cómo piensa que está la ciudad de Quito?', el 52\% de los encuestados opinó que mejor, porcentaje superior al $38 \%$ opinó lo mismo en junio del 2011; y sobre la situación del barrio en comparación a un año atrás, el 36,2\% asegura que esta es mejor, una cifra que supera al $28 \%$ registrado cuando se hizo la misma pregunta en una encuesta realizada en junio de 2011 (La Hora 2012).

Analizando los porcentajes presentados, se puede observar que Barrera, luego de tres años de gestión, incrementó su legitimidad material o del ejercicio en términos porcentuales en un $29,46 \%$, esto comparando al $43,14 \%$ de su elección respecto al $72,6 \%$ de apoyo a su gestión en 2012 , lo cual representa en término de ciudadanos un incremento de legitimidad del 68,28\%, un porcentaje nada despreciable.

El segundo caso que se analiza es el de la elección y gestión del alcalde Mauricio Rodas. Estas elecciones se llevaron a cabo el 23 de febrero de 2014, en las cuales Rodas alcanzó la alcaldía capitalina con el 58,55\% de los votos, frente al 38,01\% que alcanzó su principal contrincante, el hasta entonces alcalde Barrera, quien terciaba para lograr su reelección con base en las obras realizadas durante su período de alcalde y con el apoyo del aparataje estatal y de la figura del presidente Correa.

Pero esta vez no le alcanzó al alcalde Barrera para hacerse de la reelección, y le dio paso a Rodas quien entró a la gestión municipal acompañado de 10 de los 21 concejales, lo cual representaba un $48 \%$ de apoyo en el concejo, asegurándose, de inicio, problemas de gobernabilidad, considerando adicionalmente que los otros 11 concejales eran parte de la oposición (Alianza País). Rodas llega a la alcaldía del DMQ como la antítesis de lo que el correísmo, materializado en Barrera y su gestión como burgomaestre, había mostrado y entregado a la ciudadanía. Rodas, quien fuera antes candidato presidencial, en 2013 ya anunció su candidatura a la alcaldía, y la efectuó 
con visitas a los barrios de la ciudad, enfocándose en los defectos de la gestión de Barrera y ofreciendo a la ciudad un gobierno municipal responsable.

Con orientación de derecha, Rodas, a lo largo de su gestión experimentó serios problemas, siendo los principales el inadecuado manejo de los residuos sólidos y los problemas de movilidad - diferencias con los taxistas y la no concreción de una nueva tarifa para el transporte público- - De los aspectos positivos, el más relevante es el avance significativo para la construcción del Metro de Quito y la reorganización de la administración municipal que efectuó prácticamente en el ocaso de su período al frente de la alcaldía metropolitana.

En este contexto, a inicios de julio del año 2016, la encuestadora Perfiles de Opinión obtuvo cifras negativas con relación a la aceptación de la gestión del alcalde Rodas. En dicha encuesta, únicamente el 47\% calificaba de positiva (suma de buena y muy buena) la gestión del alcalde, quien en ese momento llevaba dos meses recolectando ayuda humanitaria para los afectados por el terremoto que golpeó de manera significativa a las provincias de Manabí y Esmeraldas. Es así como Rodas experimentó fluctuaciones en sus niveles de aceptación ciudadana durante los más de dos años que llevaba al frente de la capital. En noviembre de 2015, por ejemplo, su calificación favorable llegó al 33,4\%, una de las más bajas que ha recibido. Pero en ningún momento retomó del nivel positivo de $89 \%$ que tenía en junio de 2014, al inicio de su gestión (El Telégrafo 2016).

Estas cifras muestran que Rodas, a dos años de haber iniciado su mandato, experimentó un decrecimiento de su legitimidad material, reflejada en la pérdida de aceptación de la ciudadanía ante su gestión, que cayó del $58,55 \%$ de votos con los que ganó la alcaldía (legitimidad de origen) al 47\% de aceptación al 2016, con una reducción de 11,55\% en su legitimidad del ejercicio en términos porcentuales, pero en términos de ciudadanos cayó aún más $(20 \%)$.

La actual alcaldesa de la ciudad de Guayaquil, Cynthia Viteri, quien se posesionó el 14 de mayo de 2019 luego de que Jaime Nebot gobernara la ciudad portuaria por 19 años, llegó a la alcaldía con el 53\% de los votos válidos en las elecciones que registraron el mayor número de candidatos de las últimas cinco décadas, con un total de 17 postulantes, acompañada además por 12 coidearios de los 15 ediles electos para conformar el Concejo 
guayaquileño. Al ser aquellos del mismo partido al que pertenece Viteri, la alianza Partido Social Cristiano-Madera de Guerrero (PSC-MG) (González 2019), entra con el $80 \%$ de respaldo en el Concejo, que se traduce en una fuerte gobernabilidad.

Durante sus primero 100 días de gestión, la alcaldesa dio prioridad a temas como el Acuerdo Nacional por la Seguridad que suscribió con el Ministerio del Interior, a la salud con el inicio del Plan de Prevención y Erradicación de adicciones a las sustancias psicotrópicas y estupefacientes mediante la implementación de clínicas móviles en barrios densamente poblados por personas de escasos recursos. Sin embargo, las acciones tomadas ante la emergencia sanitaria por la COVID-19 experimentada con fuerza en la ciudad porteña desde marzo de 2020 no han sido suficientes para la mayor parte de la ciudadanía quienes, en marzo de 2020 le han dado únicamente el $21 \%$ de apoyo a su gestión, ubicándola en el último lugar (décimo) en Latinoamérica de los alcaldes analizados por la encuestadora mexicana Mitofsky (2020). ${ }^{1}$

Ante estas cifras se evidencia que Viteri, tras casi un año de haber comenzado su mandato, ha experimentado un decrecimiento considerable en los niveles de legitimidad, pasando del $53 \%$ con el que inició, al $21 \%$ en marzo, lo que representa una reducción porcentual de $32 \%$, es decir, aproximadamente 6 de cada 10 personas que votaron por Viteri, no aprueban su gestión en la alcaldía.

Estos análisis verticales y horizontales han permitido contextualizar lo que a continuación se revisará, al observar en qué condiciones de aceptación ciudadana llegó Yunda a la alcaldía capitalina, y cómo, luego de casi un año se encuentra posicionado en el cargo de burgomaestre gracias a ciertos factores que también trataremos de sistematizar.

1. La encuesta mide el estado de la aprobación ciudadana en varios países, realizando una recopilación de encuestas publicadas en medios electrónicos. 


\section{Gestión del alcalde Yunda en su primer año de gobierno}

\section{La legitimación continúa en acción}

Jorge Yunda Machado, un guaneño de 55 años, es el actual alcalde de la capital ecuatoriana. Se considera a sí mismo hiperactivo, buscando siempre algo que hacer, es así que es médico de profesión, pero también músico, locutor y empresario (Carvajal y Márquez 2019). Antes de ser candidato a la alcaldía, fue asambleísta. Yunda aprovechó de manera importante su capital social, como figura pública, al momento de oficializar su candidatura a la alcaldía de Quito. Es así como:

La campaña del alcalde Jorge Yunda centró sus lineamientos en tres ideas generales que giraban en torno a la trayectoria personal, profesional y ciudadana de este, las cuales son: Jorge en la música, Jorge en la Asamblea, Jorge el animalista y Jorge el ciudadano. (Calderón y Jaramillo 2019, 203)

Como dueño de medios de comunicación, Yunda también aprovechó su experticia en este ámbito para montar una plataforma importante en los medios de comunicación y redes sociales, uno de los puntales de su campaña y carrera hacia la alcaldía capitalina (Calderón y Jaramillo 2019), lo que supo aprovechar en un contexto político nuevo, con una ausencia sentida de una figura como Correa que durante los últimos 10 años había absorbido cualquier aspiración diferente a él, lo que "produjo una verdadera primavera de nuevos brotes políticos y ciudadanos que dio como resultado más de trecientos movimientos y partidos políticos" (Vega 2019, 26) a nivel nacional.

Jorge Yunda ganó la alcaldía del DMQ con apenas el 21,30\% de los votos, la votación más baja en 27 años de elecciones capitalinas (Quiñónez 2020), compitiendo además con la cifra récord de 17 candidatos más, superando a Luisa Maldonado de la línea correista que obtuvo el 18,42\%, a Paco Moncayo con el 17,78\% y a César Montúfar con el 16,93\%, en una apretada elección, donde todos los demás candidatos obtuvieron menos del 10\% de los votos válidos. Adicionalmente, Yunda llegó con únicamente 3 ediles de los 21 posibles, un hecho que de incio marcaría su dificultad para materializar una gobernabilidad plena en su gestión como burgomaestre, sumada a la ya mencionada escasa legitimidad de origen o formal. 
Durante los primeros meses, el alcalde Yunda tomó acciones como la venta de autos municipales y una reducción considerable de servidores públicos del municipio, la extención del pico y placa a todo el día con el programa denominado "Hoy no circula" y la repavimentación de una cantidad importante de tramos viales de alto flujo en la ciudad, aunque criticado por el exalcalde Roque Sevilla quien manifestó en su momento que estas acciones de repavimentación ya no son trascendentes, que lo importante actualmente es ver qué pasará con la movilidad en su conjunto, como por ejemplo con el metro de la ciudad, comenzando con un factor crucial, como quién lo va a administrar (Sevilla, en Quiñónez 2020).

Quizás el momento más duro que ha tenido que experimentar el alcalde Yunda fue asumir el liderazgo capitalino durante las manifestaciones del pasado octubre de 2019, cuando a la capital llegaron miles de manifestantes de todo el país contra las medidas del gobierno en relación al subsidio de los combustibles. En esos momentos de conflicto social, el alcalde mantuvo un bajo perfil, y no actuó en relación a la relevancia de lo sucedido (Quiñónez 2020). No es casual que concluido el primer semestre de gestión, el mismo alcalde Yunda se autocalificara con un 3 sobre 10, siendo posiblemente algo exigente en su apreciación (Burbano 2020).

Sin embargo, es importante destacar el hecho de que, un mes antes a los acontecimientos, Yunda se constituía como el quinto mejor alcalde en latinoamérica, cuando la encuestadora mexicana Mitofsky, quien realiza una encuesta semestral, emitió un informe con los resultados de aprobación ciudadana de 13 ciudades latinoamericanas, en el cual Yunda aparece como el quinto mejor alcalde, con el $53 \%$ de aprobación de la gestión (Mitofsky 2019). La lista está encabezada por David Collado de Santo Domingo con el $85 \%$, Claudia Sheinbaum de la Ciudad de México con el 59\% y Horacio Rodríguez de Buenos Aires con el 59\%.

Es así como llega 2020, y con él una de las crisis más importantes de los últimos 100 años, solo comparable a la de 1929 en la caída de la Bolsa de Nueva York (BBC 2020) y todo lo que eso representó para la humanidad la pandemia por la COVID-19 que ha paralizado al mundo entero y ha matado, hasta el momento, a centenas de miles de personas alrededor del globo. En este contexto, el alcalde Yunda desde marzo de 2020, cuando en el Ecuador se empiezan a registrar contagios y muertes, se ha comprometido de forma 
personal en atender y paliar la crisis, inclusive llegando a donar la totalidad de su sueldo para aportar en este duro momento (Carvajal 2020).

Es así como Yunda se convierte en el segundo alcalde con más aceptación en Latinoamérica, con un $89 \%$, superado únicamente por David Collado de Santo Domingo, que al finalizar su cargo logra una aprobación de $93 \%$, y en tercer lugar está Andrew Coumo de Nueva York con el $84 \%$, que es el Estado de Estados Unidos a la fecha con mayor número de infectados por la COVID-19 y muertes (Mitofsky 2020). Como se ha mencionado, la legitimidad "es un fenómeno interactivo que se origina en el consentimiento que la ciudadanía otorga a los candidatos en las elecciones, pero que se ratifica o se rechaza en las evaluaciones del rendimiento que hagan de las autoridades una vez que asumen sus cargos" (Prado 2010, 8).

El factor de cercanía, que fue mencionado al inicio de este documento, es también fundamental. La legitimidad, entendida como de proximidad o identidad, se alimenta de la cercanía de los mandatarios a sus mandantes y, definitivamente, mientras los gobiernos nacionales están más alejados de los ciudadanos, por el otro lado están los gobiernos municipales, más cercanos al pueblo y con la posibilidad de mostrar más efectivamente sus acciones, especialmente a aquellos que viven en ese municipio (Prado 2010). Pero se necesita algo más que cercanía, se trata de ejercer lo que se denomina como la democracia continua, resultado de la mutación o evolución de las democracias actuales, que exigen a los mandatarios ganarse la confianza de sus votantes cada nuevo amanecer (Cheresky 2012).

Prueba de que estamos ante una democracia continua y evolucionada, son las diferencias entre las acciones que han realizado Viteri en Guayaquil y Yunda en el DMQ, con sus respectivos resultados. Mientras Viteri inició su gobierno con una votación de $53 \%$, en los actuales momentos y con más deudas que aciertos en el manejo de la crisis sanitaria, con un Guayaquil puesto de referencia a nivel latinoamericano como lo que no se debe hacer en materia de administración pública municipal, ha llegado a niveles de aceptación del $21 \%$, mientras Yunda y sus acciones han hecho que luego de ganar las elecciones con apenas un quinto de los votos, llegue a casi el 90\% de aceptación. 
Figura 1

Comparativo de la opinión ciudadana de las gestiones de Yunda y Viteri 2019-2020

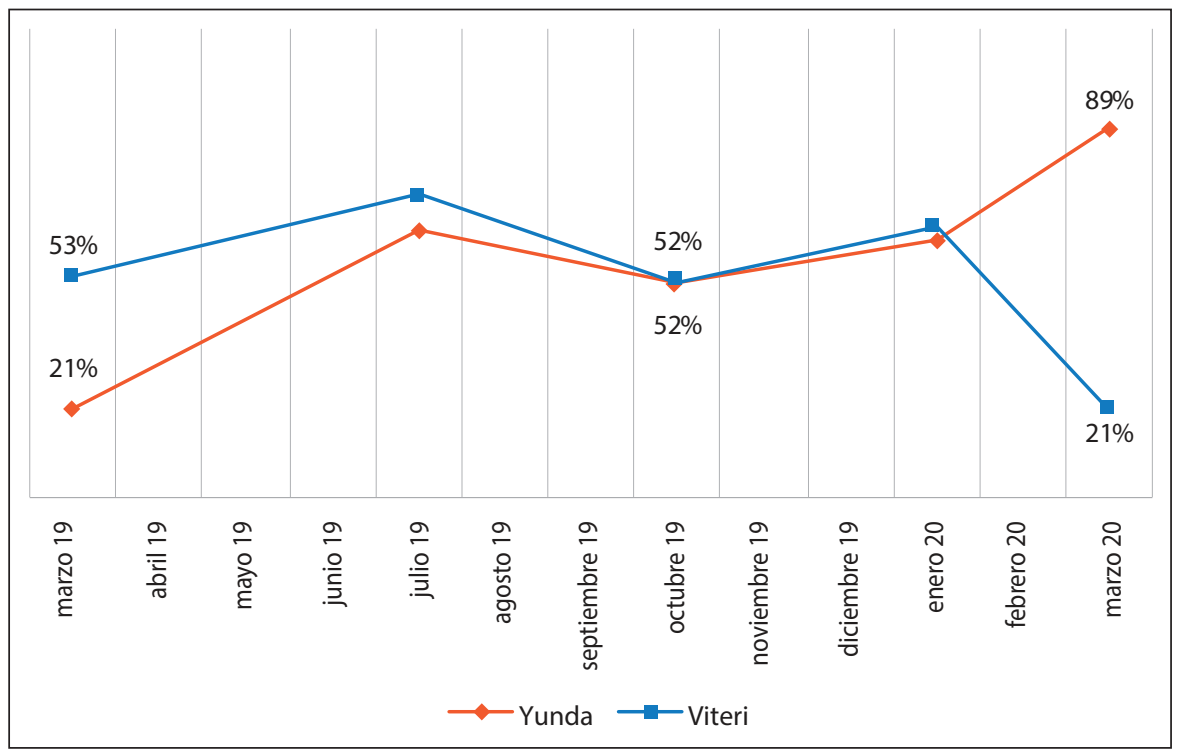

Fuente: Mitofsky (2019; 2020); Perfiles de Opinión (2020).

El análisis se repite al comparar la gestión de los alcaldes Barrera y Rodas, con la de Viteri y Yunda. Barrera obtiene una diferencia positiva de 29\% al confrontar su legitimidad de origen versus la del ejercicio; en cambio, Rodas refleja un - $12 \%$ en esta misma comparación. Viteri es la menos favorecida del grupo con un $-32 \%$, y Yunda tiene $68 \%$ más de aceptación en los actuales momentos respecto a su legitimidad inicial que fue del $21 \%$, a lo que hay que añadir que este incremento se presenta en medio de una crisis sanitaria mundial. 
Figura 2

\section{Diferencias porcentuales entre legitimidad de origen y del ejercicio de los alcaldes analizados}

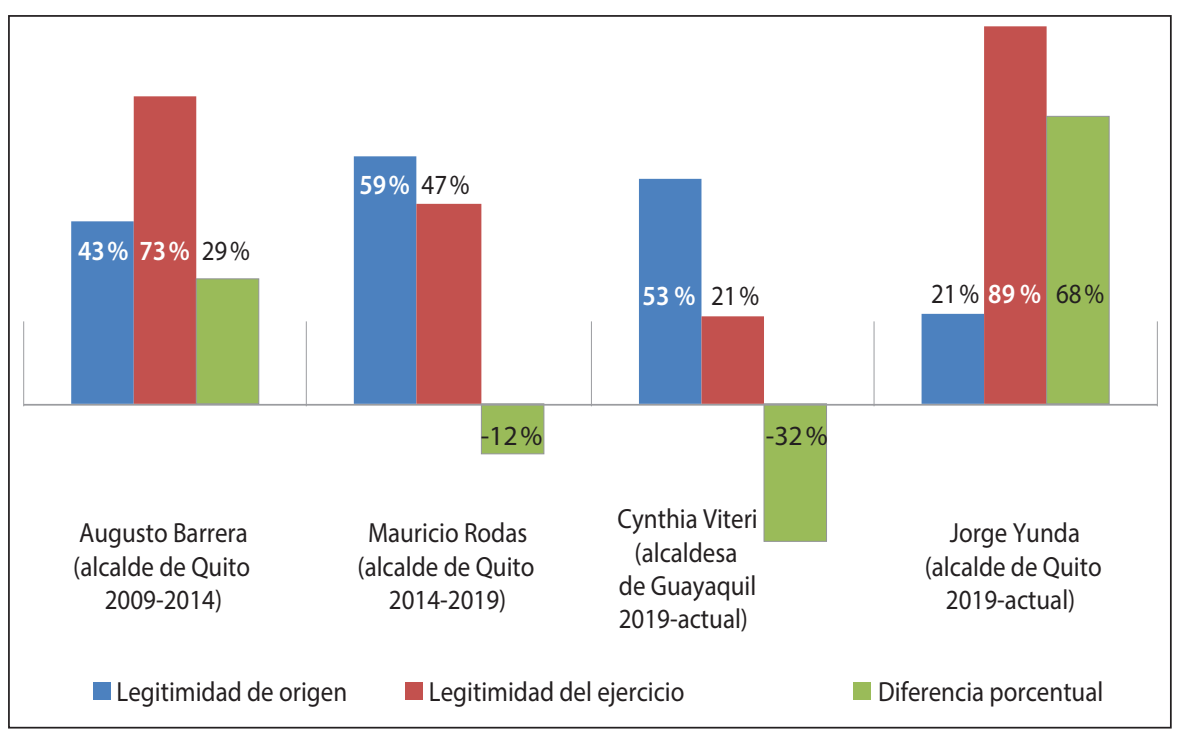

Fuente: Mitofsky (2019; 2020); Perfiles de Opinión (2020).

Este análisis porcentual, de la diferencia entre las dos legitimidades —de origen y del ejercicio-, es muy diciente; más aún al analizar el nivel de variación, de los crecimientos o decrementos porcentuales de la legitimidad del ejercicio en función de la de origen. Barrera experimentó un crecimiento del $68 \%$, Rodas un decrecimiento del $-20 \%$, Viteri un $-60 \%$, y Yunda un aumento de su legitimidad del ejercicio respecto a la de origen en un $321 \%$, es decir, cuadruplicó el número de personas que confían en su gestión. 
Figura 3

\section{Diferencias porcentuales en función de la legitimidad de origen de alcaldes analizados}

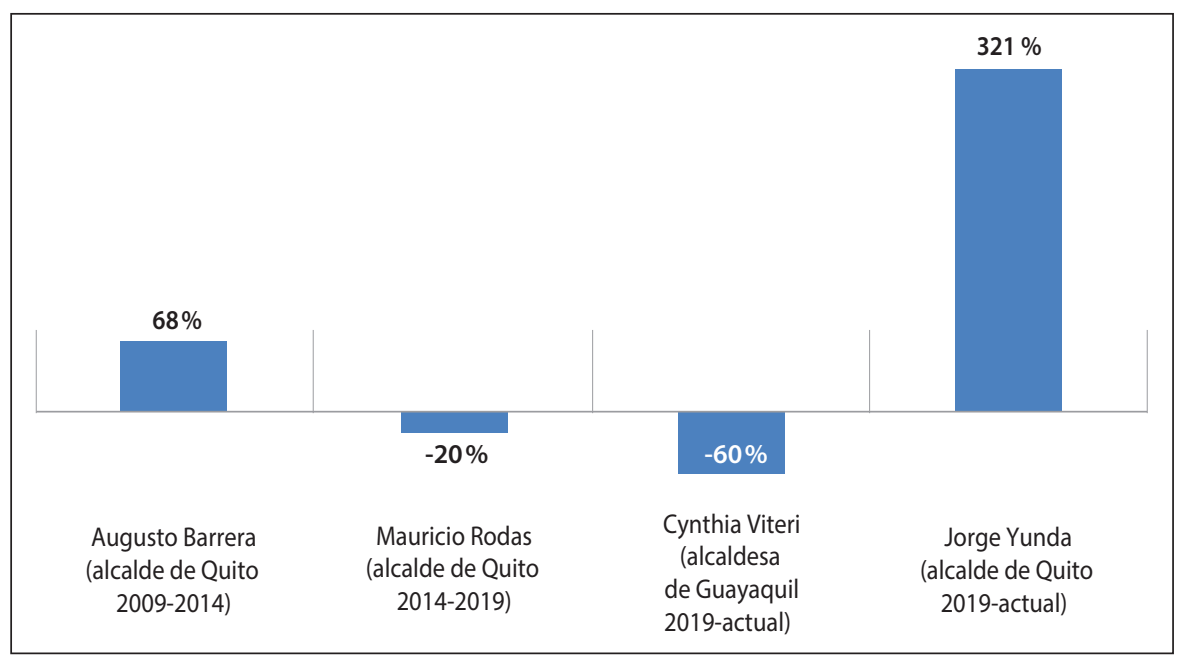

Fuente: Mitofsky (2019; 2020); Perfiles de Opinión (2020).

Los datos son corroborados por la encuestadora Cedatos (2020), que publicó en su portal, el 17 de abril, los niveles de aprobación o desaprobación a la gestión de varias autoridades e instituciones frente a las crisis de la COVID-19. Las respuestas fueron: aprobación del alcalde Yunda con el $82 \%$, siendo el más alto con respecto a los diferentes niveles de gobierno; aprobación de la alcaldesa Viteri con el 32\%; aprobación del alcalde Pedro Palacios de Cuenca con el 48\%; aprobación del presidente de la República Lenín Moreno, con el 24\%.

¿A qué se debe tanta credibilidad y nivel de aceptación del alcalde Yunda en tan poco tiempo? Sin duda un factor crucial ha sido la respuesta que ha tenido ante la crisis sanitaria, es posible que Sartori $(2003,161)$ tenga razón cuando expresó que "los políticos son populares en tiempos heroicos, pero pocas veces lo son en los tiempos rutinarios, cuando la política de la democracia se convierte en un confuso y ordinario esfuerzo diario". 
Sin embargo, el fenómeno Yunda viene en sostenido crecimiento desde el año pasado luego de superar el bache en su gestión durante las manifestaciones de octubre del 2019. Son varios los aspectos destacados en el desempeño del actual alcalde que han sido sistematizados a partir de una entrevista a Rogelio Echeverría, coordinador de la Unidad Especial Regula tu Barrio en Quitumbe y Eloy Alfaro:

Tabla 1

Factores de éxito de la administración Yunda al mando del DMQ

\begin{tabular}{|c|c|}
\hline Factor de éxito & Descripción \\
\hline Confianza & $\begin{array}{l}\text { La gestión del alcalde Yunda ha ido sumando confianza en la ciu- } \\
\text { dadanía con el pasar del tiempo, llegando al punto de que hace } \\
\text { pocos días decidió donar su sueldo para ayudar a los ciudadanos } \\
\text { en el contexto de la crisis sanitaria por la COVID-19. }\end{array}$ \\
\hline Cercanía con la gente & $\begin{array}{l}\text { El alcalde Yunda visita constantemente los barrios, escucha de las } \\
\text { propias personas sus necesidades y trata de atenderlas en el mar- } \\
\text { co de las competencias municipales. Sumado a esto, está el hecho } \\
\text { de que las nueve administradoras zonales son mujeres, con lo cual } \\
\text { trata de dar un enfoque de género a su gestión. }\end{array}$ \\
\hline Asesorarse por expertos & $\begin{array}{l}\text { Al autorreconocerse como una persona inexperta en gestión públi- } \\
\text { ca, desde el inicio de su administración se ha rodeado de personas } \\
\text { especializadas en materia de política urbana y planificación, como } \\
\text { es el caso de Fernando Carrión, reconocido experto en los temas } \\
\text { de ciudad, quien es uno de sus principales asesores. }\end{array}$ \\
\hline Excelente manejo de medios & $\begin{array}{l}\text { Si de algo sabe el alcalde Yunda es de medios de comunicación, } \\
\text { ya que es dueño y trabajó hasta antes de posesionarse en el cargo } \\
\text { en diversos medios de radio y televisión. }\end{array}$ \\
\hline $\begin{array}{l}\text { Gobierno abierto y gobierno } \\
\text { electrónico }\end{array}$ & $\begin{array}{l}\text { Ha implementado plataformas web como la de registro de nece- } \\
\text { sidades para prestar ayuda a la ciudadanía, de forma especial en } \\
\text { el contexto de la COVID-19. También impulsó la Radiotón para } \\
\text { recaudar fondos para el propósito antes mencionado, entre otras } \\
\text { acciones que involucran de forma directa a la ciudadanía. }\end{array}$ \\
\hline Proactividad & $\begin{array}{l}\text { Su gestión se caracteriza por anticiparse a los problemas, tratado } \\
\text { de prevenirlos y mitigarlos. }\end{array}$ \\
\hline
\end{tabular}

Fuente: adaptado de Echeverría (2020). 
Los elementos rescatados por Echeverría (2020) muestran que, aunque los gobernantes pueden tener problemas de gobernabilidad de origen, como la falta de experiencia en gestión pública, baja legitimidad reflejada en las urnas o momentos de inacción ante situaciones inesperadas como los conflictos sociales, la actitud y la motivación son determinantes para afrontar retos de envergadura como la administración del DMQ.

\section{Conclusiones}

El régimen político ecuatoriano se encuentra en transición desde hace algún tiempo. El punto de partida que se puede identificar es cuando acabó la influencia directa del expresidente Correa en el actual mandatario, Lenín Moreno, el cual se manifestó a mediados de 2017, cuando Moreno da un giro inesperado a su política, pasando de un declarado socialismo del siglo XXI, a un centro derecha con tintes neoliberales.

En este contexto de extrañeza política, respecto a lo experimentado en el país en la última década, el sistema de partidos que, con la era correísta había quedado sepultado, comenzaba a desenterrar viejas usanzas de hacer política y de satisfacer intereses particulares. Es así como se le presenta la oportunidad a un político poco conocido hasta entonces, Jorge Yunda, que únicamente había ostentado el cargo de asambleísta, pero que en la contienda seccional de inicios de 2019 aspiraba a la alcaldía de la ciudad más importante del Ecuador.

Con muy pocas esperanzas en las encuestas previas a la elección, Yunda se fue ganando de a poco un espacio entre el top cinco de los 17 candidatos que terciaban las elecciones $\mathrm{y}$, a través de una campaña manejada mediáticamente con mucha capacidad, aupada a la experiencia y poder comunicacional, sin olvidar el gran esfuerzo realizado en los barrios sobre todo del centro y sur de la capital, Yunda gana con un margen muy estrecho las elecciones, lo cual, evidentemente, le dejó por delante un gran reto: consolidar su legitimidad de forma continua, en el día a día, con la gente.

Yunda ha logrado su propósito, con altibajos (como todo ejercicio administrativo público), pero sobre todo con una clara determinación de mejorar 
su legitimidad respecto a cómo arrancó su gestión. Le quedan aún tres años de gestión, y muchas cosas pueden pasar, pero este estudio indudablemente muestra que en este primer año Yunda ha demostrado que su legitimidad del ejercicio ha eclipsado bastante bien a la escasa legitimidad con la inició su mandato como alcalde de la capital de todos los ecuatorianos.

\section{Referencias}

Annunziata, Rocío. 2011. "La política de la sigularidad de la experiencia". En Ciudadanía y legitimidad democrática en América Latina, compilado por Isidoro Cheresky, 395-444. Buenos Aires: Editorial Prometeo.

BBC. 2020. "Coronavirus y economía: cómo fue la Gran Depresión con la que comparan el impacto económico de la pandemia". Accedido noviembre. https://cutt.ly/YgKNOH0.

Burbano, Fernando. 2020. "Yunda: 3/10". En El Universo. Accedido enero. https://cutt.ly/ vfYAjHs.

Calderón, María, e Ismael Jaramillo. 2019. "Mensaje político y eficacia electoral: microsegmentación en redes sociales en las elecciones seccionales de Quito". En Democracias, editado por Isidro Cheresky, 179-210. Quito: Instituto de la Democracia. https://bit.ly/2F tOxXK.

Carvajal, Ana. 2020. "El alcalde Jorge Yunda donó su sueldo para radiovirtualmaratón Quito Solidario". El Comercio. Accedido marzo. https://cutt.ly/QgK1yLC.

Carvajal, Ana, y Cristina Márquez. 2019. "Quito, en manos de un médico, locutor y músico". El Comercio. Accedido mayo de 2020. https://cutt.ly/VgLEzp8.

Cedatos. 2020. "El covid-19 cambió la vida y expectativas de los ecuatorianos. Apreciaciones de la población al 16 de abril de 2020". Accedido abril. https://cutt.ly/QfYATKA.

Cheresky, Isidro. 2012. "Mutación democrática: otra ciudadanía, otras representaciones". En ¿Qué democracia en América Latina?, editado por Isidro Cheresky, 23-54. Buenos Aires: CLACSO / Prometeo libros.

Duverger, Maurice. 1957. Los partidos políticos. Ciudad de México: FCE.

--- 1962 . Instituciones politicas y derecho constitucional. Madrid: Ariel.

EC. 2008. Constitución de la República del Ecuador. Registro Oficial 449, 20 de octubre.

El Telégrafo. 2016. "La aceptación de Rodas cayó 7,6 puntos entre junio y julio". El Telégrafo. 11 de julio. Accedido abril de 2020. https://tinyurl.com/yxzm6lqc.

Freidenberg, Flavia. 2011. "Ecuador 2009: las elecciones que consolidan el cambio del sistema de partidos". En América Latina: política y elecciones del bicentenario (2009-2010), editado por Manuel Alcántara y María Tagina, 63-99. Madrid: Centro de Estudios Políticos y Constitucionales. 
Galindo, Adrián. 2016. Legitimidad en los gobiernos latinoamericanos durante la primera década del siglo XXI. Un comparativo entre México, Argentina y Brasil. Ciudad de México: UAM.

García, Roberto. 2000. "Fundamentos de la legitimidad". Estudios Políticos 24: 129-153.

González, Jorge. 2019. "Cynthia Viteri gobernaría Guayaquil con una mayoría en el Concejo, según escrutinio del CNE”. El Comercio. Accedido marzo de 2020. https://cutt.ly/ AfYAXgu.

Hernández, Edmundo. 2004. "Gestión financiera y legitimidad municipal”. Revista Eletrônica de Ciência Administrativa (2): 1-11.

La Hora. 2012. "Según encuesta, Augusto Barrera mantiene elevados niveles de credibilidad y aprobación”. Diario La Hora. Accedido julio de 2020. https://www.lahora.com.ec/noticia/ 1101356522/noticia.

Linz, Juan. 1991. La quiebra de las democracias. Buenos Aires: Alianza.

Lipset, Seymour. 2001. "Algunos requisitos sociales de la democracia: desarrollo económico y legitimidad política”. En Diez textos básico de ciencia política, editado por Albert Bat1le, 112-150. Barcelona: Ariel.

Malamud, Andrés. 2003. "Partidos políticos". En Introducción a la ciencia política, editado por Julio Pinto, 321-350. Buenos Aires: Eudeba.

Mitofsky. 2019. "Aprobación de alcaldes de América”. Mitofky. Accedido abril de 2020. htt ps://cutt.ly/6fYSEMk.

---. 2020. "Aprobación de alcaldes de América. Expertos analizan la gestión del alcalde Jorge Yunda”. Accedido abril. https://cutt.ly/5fYAVI6.

Moncagatta, Paolo, Arturo Moscoso y Simón Pachano. 2020. Cultura política de la democracia en Ecuador y en las Américas, 2018/19: tomándole el pulso a la democracia. Quito: USAID.

OCDE. 2020. Panorama de las administraciones públicas en América Latina y el Caribe 2020. París: OCDE.

Payne, Mark, y Andrés Allamand. 2006. La politica importa: democracia y desarrollo en América Latina. Washington D. C.: BID.

Perfiles de Opinión. 2020. "Calificación a la gestión del alcalde Jorge Yunda y Cynthia Viteri”. Perfiles de Opinión. Accedido abril. https://cutt.ly/FfYAM6m.

Prado, Silvio. 2010. Legitimidad municipal. Managua: CEAP.

Quiñónez, Hellen. 2020. “Expertos analizan la gestión del alcalde Jorge Yunda”. Noticiero 24 Horas en Teleamazonas. 2 de enero. Accedido abril. https://cutt.ly/2fYA24G.

Rúa, Carlos. 2013. "La legitimidad en el ejercicio del poder político en el estado social de derecho. Una revisión desde el caso colombiano". Ius et Praxis (2): 85-122.

Sartori, Giovanni. 1995. Teoría de la democracia. Madrid: Alianza.

---. 2003. Ingeniería constitucional comparada. Ciudad de México: FCE. 
Vairo, Daniela. 2012. “El 'consenso de los perdedores' y la legitimidad de la democracia en América del Sur”. Política y Gobierno (1): 41-69.

Vallès, Josep. 2015. Ciencia política: un manual. Barcelona: Planeta.

Vega, Fernando. 2019. "Primeras reflexiones: elecciones 2019”. Sociología y Política Hoy (1): 26-30.

Weber, Max. 2002. Economía y sociedad. Madrid: FCE.

Wikipedia. 2020. "Viteri, Cynthia". Accedido abril. https://es.wikipedia.org/wiki/Cynthia_Vi teri.

\section{Entrevista realizada por Guido Moncayo}

Echeverría, Rogelio. 2020. Coordinador de la Unidad Especial Regula tu Barrio en Quitumbe y Eloy Alfaro. 